\title{
CBTC (Communication Based Train Control): system and development
}

\author{
N. Bin, T. Tao, Q. K. Min \& G. C. Hai \\ Department of Control Engineering, \\ School of Electronics and Information Engineering, \\ Beijing Jiaotong University, Beijing, P. R. China
}

\begin{abstract}
CBTC (Communication Based Train Control) systems are known as comprehensive, integrated and intelligent control systems for rail systems including mainline railways, light rails and underground lines in cities. With the development of modern data communication, computer and control techniques, CBTC represents the future direction of rail control systems. At present, CBTC has been used in light rail and underground lines in cities. It has not been implemented in mainline railways for many reasons. In future decades, rail systems will be in rapid development periods throughout the world. CBTC is known as the brain and nerve centre of rail systems, and ensures the safety and efficiency of rail systems. It is necessary for CBTC to be researched and developed further. In Europe, there is the ETCS (European Train Control System). In China, there is the CTCS (Chinese Train Control System). In Northern American and Japan, there are advanced train control systems or moving block systems. However, there is no standard for development and design of CBTC in the world at present. In this paper, efforts are made towards the establishment of a CBTC standard which directs the development and design of CBTC systems. The configuration of CBTC systems is first described. The key technical issues are addressed. The fundamental modular of CBTC and its interface requirements are defined. The transit methods from the present train control systems based on track circuits, transponders and other traditional means to CBTC systems are also put forward.
\end{abstract}

Keywords: automation control system, rail system, computer and communication, standardization. 


\section{Introduction}

The CBTC (Communication Based Train Control) system has been known as the development direction of control systems for rail systems in the world. In particular, with quick development of modern mobile communication, its implementation and application become more and more easily. At present, CBTC has been used in city rail transportation systems, such as light rail and underground systems. It will be used in mainline railway systems in the near future. Application of CBTC has the following features. It makes the dispatching system more flexible and efficient. The safety and reliability of the system are high. It is easy for CBTC to be transited from the present system. The maintenance cost for CBTC is lower since track circuits are removed from the system. The control system of railway network will be towards intelligent, network and comprehensive system, CBTC is as brain and nerve centre of the railway system. Its development and application will be with the direction of railway operation control system [2].

At present, there are CBTC systems from the different company in the world. For example, SELTRAC from Alcatel has been used in SkyTrain in Vancouver in Canada since 1986, in JFK-Airport Light Rail System in the United States since 2003. Trainguard MT from Siemens will be used in the underground line Canarsie in New York in 2006. URBALIS from Alstom has been used in the Light rail in Singapore since 2003. In addition, there are also CBTC systems from Japan Signaling, GE and CSEE etc. In the world, there are more than 30 light rail and underground systems where CBTC systems have been or will be applied. According to the statistics, most of the CBTC systems are based on cross-loops for train-ground communication, some of the CBTC systems use radio for train-ground communication. In Europe, Radio system will be used for train control (ETCS-2 and Euro-radio) [1]. Nowadays, when the signaling systems need to be upgraded in many cities, such as London, Paris and New York, CBTC system is chose as a new system. It is predicted that CBTC systems will be applied in mainline railway in the near future [5].

However, there is no standardization for CBTC systems. The CBTC systems from the different company cannot be compatible. It is easy for a kind of CBTC system from a company to monopoly the market. It is not good for commercial competition and technical development. For users of a railway network, it is not possible to select the best systems. Meanwhile, it is not easy for the CBTC system to be upgraded with technical development. In the paper, efforts are made to put forward the technical standardization for CBTC in terms of system configuration, function requirements, dada format, interface definition and development in order to facilitate development and design of CBTC systems. Meanwhile, CBTC systems from the different companies can be compatible on a railway network and railway users have more choices in the CBTC markets. Of course, for every designer of a CBTC, it is easier for the whole system or part of the system of a CBTC to be upgraded with new technology advent. 


\section{System configuration of CBTC}

A CBTC system can be divided into the five parts. The first part is the Central Control System (CCS). The second part is the Station Control System (SCS). The third part is the Onboard Control System (OCS). The fourth part is the Block Control System (BCS), including Radio Block Control System (RBCS) and block sensors etc. The fifth part is the Communication Network System (CNS), including mobile communication system. The figure 1 shows the configuration of a CBTC system. The five parts constitutes the whole system of a CBTC system. However, the five parts are relatively an independent part each other as a subsystem of a CBTC. The fifth part (CNS) connects all other four parts as communication channel, including mobile communication between train and wayside systems in order to ensure real-time, reliable and safe data exchange among them. The functions of a CBTC system should be distributed to the five subsystems. The data format between every two parts should be defined.

The Central Control System (CCS) is the control center of a CBTC system. Train plans and train graphs are generated here. All the train operation is dispatched and commanded in CCS according to train graphs. The state data concerning to station control system (SCS), block control system (BCS), onboard control system (OCS) and train operation should be sent to CCS [1].

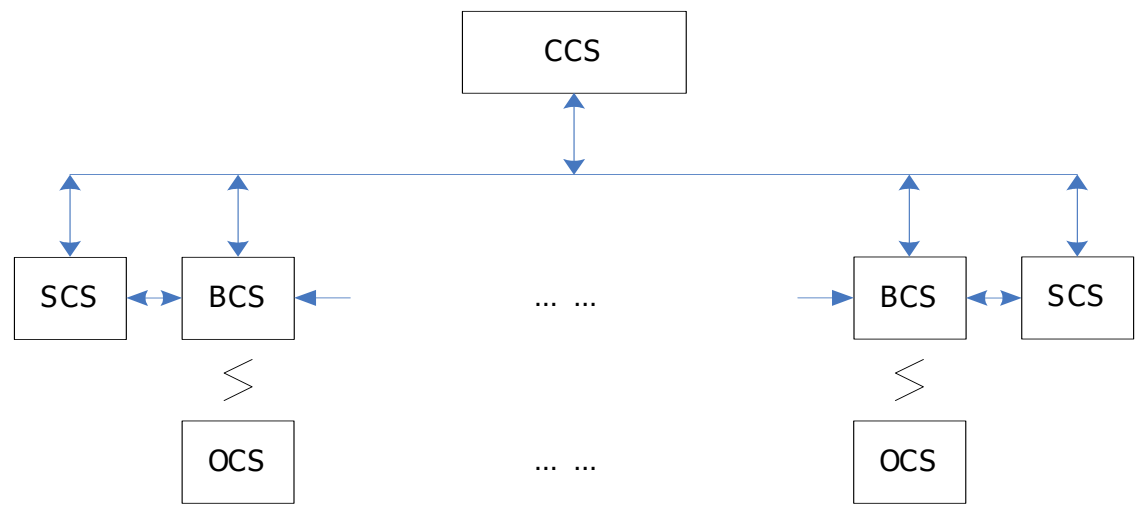

Figure 1: The configuration of CBTC systems.

Station Control System (SCS) is an interlocking system which control switches, signals and routes at stations or in areas. SCS communicate with CCS, BCS and OCS. Station interlocking system is a traditional train operation control system in terms of its functions. There is no special requirement for SCS in CBTC systems. At present, most of SCS are the computer based interlocking systems in CBTC systems [2].

Block Control System (BSC) includes radio block system and wayside sensors for CBTC system along the track. BSC communicate with CCS, SCS and OCS. Its main function is to control train safety operation in blocks. Train operation safety interval is calculated by BCS according to train safety operation 
modes on moving block conditions, and then the concerning orders (permissive speed) are sent to OCS by CNS.

On-board Control System (OCS) is a train control system equipped in locomotives. Its main function is to control train speed such as acceleration, deceleration, cruising and braking. Data concerning train position and speed are sent to BCS. The permissive speed of train operation is received by OCS [3]. In a CBTC system, OCS must be safer and more intelligent compared with OCS in the traditional train control system.

SCS, BCS and OCS must be failsafe and reliable. CNS must be satisfied with the requirements of real time, safety and reliable data transmission in CBTC systems. For CCS, there is no failsafe requirement since it does not directly control train operation, but it is reliable [3].

As a comprehensive train operation control system, it is reasonable for a CBTC system to be divided into the five subsystems. Every subsystem is relatively independent and easier to be designed and implemented. The above division is very helpful for a CBTC system to be analyzed, designed and developed.

\section{The key technical issues and interface requirements of CBTC}

In a CBTC system, the key technical issues can be described as follows. Some of the key technologies are common in every subsystem. The others are relatively independent.

The vital computer is one of the core parts in CBTC systems. In SCS, BCS and OCS, there is a vital computer. From the point of view of application, the vital computer is different with the different subsystems. However, in terms of safety and reliable requirements, the safety platform of the vital computer in the different subsystems is the same. The vital computer can be designed as a series modes which are suitable for different subsystem in a CBTC system. The configuration of a vital computer can be two out of two or two out of three where fault-tolerant design is carried out. The safety platform is transparent to the application in the different subsystems. The vital computer is relatively independent parts in CBTC system. Its configuration and software platform can be upgraded with technical development of computer and fault-tolerant design [6].

Figure 2 shows the configuration of an OCS in a CBTC system. The vital computer is the core part of OCS. Other parts (I, II, III, IV ...) are connected to the vital computer by a kind of bus (Can bus or other filed bus). Other parts could be radio receiver and transmitter unit, position unit, speed measurement units, MMI unit, locomotive engine interface, recorder unit etc [3]. It is obvious that OCS is modular configuration, in addition to the vital computer.

The reliable and safety mobile communication system is the foundation of a CBTC system. The concept about moving block system (CBTC is also called as moving block system) was put forward in 1960. It is not possible for a CBTC system to be implemented until the reliable, safe mobile communication appears. 
Traditional track circuits cannot satisfy with the communication requirements of train and wayside in a CBTC. In the last decades, cross-cable and leakage cable or leakage optical fiber can be used in CBTC systems. Today, GSM-R and other radio system begin to be applied in a CBTC system. The reliable and safety mobile communication system become the key technology of a CBTC system.

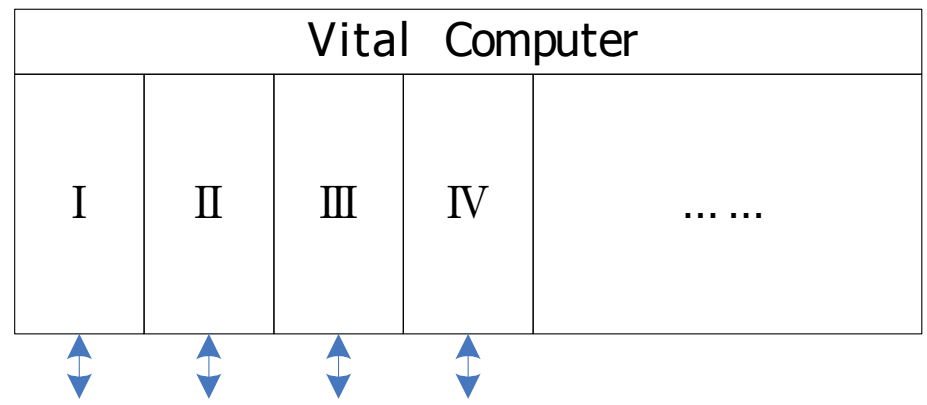

Figure 2: On-board control system configuration.

The accurate position system and the accurate speed measurement system are also the key technologies of CBTC systems. The accurate position and speed is the basic parameters of train operation control. In the position system, position calibration must be considered. In the speed measurement system, it is possible for combination of the different speed sensors (radar and axle generator) to be used to be suitable for the different speed of the train [3].

Train integrity system is very important unit in a CBTC system since track circuits are removed. An axle-counting system or on-board train integrity unit are applied for train integrity checking in a CBTC system.

The dispatching algorithms are the core software in CCS of CBTC systems. Its task is to generate train graphs according to the requirements of train plan and to automatically restore normal train operation when train operation plan is disturbed.

The train operation control model is the key to ensure train to be safe operation in CBTC systems. In a CBTC system, there is no block section. Train following interval is calculated in real time method. The figure 3 shows the principle of train following interval control in CBTC systems. In addition to the safety protection distance $\left(\mathrm{d}_{\mathrm{f}}\right)$, the interval of the two following trains (train 1 and train 2) is the safe braking distance of train 2 in theory. $V_{2}(d)$ is the speed curve of the following train $2 . \mathrm{V}_{2 \mathrm{e}}(\mathrm{d})$ is the emergency braking curve of the train 2 , and $\mathrm{V}_{2 \mathrm{~s}}(\mathrm{~d})$ is the service braking curve of the train $2 . \mathrm{O}$ is the calculated stop point of train 2. O' and O', are respectively the actual stop point of train 2, caused by the various errors. It is obvious that train operation control mode is the algorithm of train speed and interval control. Cellular Automata model is the newest model for train operation control [4].

Simulation and test platform is necessary for CBTC system development. Since CBTC system is a complicated and comprehensive system, during the development of a CBTC system, it needs a simulation environment to support 
the development. After the CBTC system is implemented and put into operation, it needs a simulation platform to test and maintain it.

\section{Development, implementation and transit}

CBTC is a comprehensive system over a rail network. The following principles should be observed in the development and design of a CBTC system.

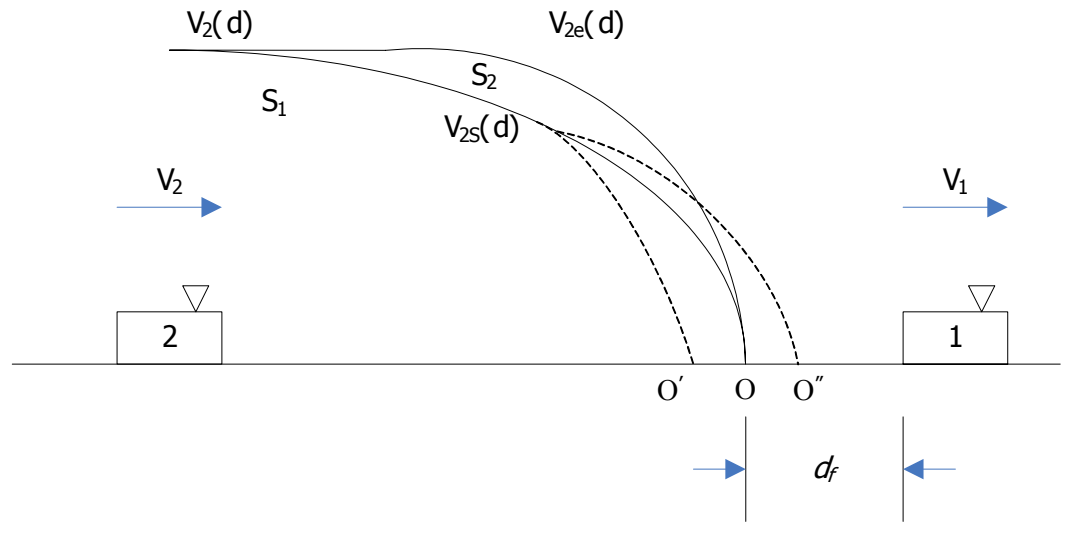

Figure 3: Train Interval control model.

According to the requirements of mainline railway network in an area or a country, light rail network or underground network in a city, the system requirements specification (SRS) and system functions specification (SFS) must be made up. These are the first set of files for a CBTC system, and they are also the basic files for a CBTC system. The files could be different for new lines and old lines upgrading in a network. This is the first step of the CBTC system development.

The second step is the key technical selection of a CBTC system. It includes the communication technology between train and wayside, the type of the vital computer and the system configuration. For communication system between train and wayside, it could be GSM-R, 2.4GHz Direct Sequence Spread Spectrum (DSSS or Frequent Hoping Spread Spectrum-FHSS) system in IEEE802.11 or cross-cable system etc. For the type of vital computer, there are more choices. It could be two out of two system or two out of three systems based on the different commercial computers. Decision on the above technologies is closely related to the cost and reliability of the CBTC systems.

Interface standardization and data format standardization should be defined before the system design. Firstly, the interfaces between CCS and SCS, CCS and BCS, SCS and BCS, OCS and BCS are defined. The dada format transmitted among the subsystems and their contents are defined. Development of each subsystem will be relatively independent and the whole system will be modular. For users, the configuration of the CBTC system will be flexible. 
After the above design files are finalized, system design and development can be started. Files management and design steps must be line with the requirements of software engineering and safety assessment procedures since a CBTC system is a safety and reliable system. Design and development for subsystems can be carried out in parallel. The chief designer must coordinate the progress of each subsystem [6].

In order to promote and verify the design and development, simulation test will be carried out in the whole process of the design. Normally, a general simulation environment platform is established to test each subsystem. Finally, after the whole system is finished, it should be simulated in laboratory to test its functions and safety features in varied conditions before it is installed in the field.

Before a CBTC system is put in operation, field test must be carried out since the simulation test in laboratory cannot be perfect. Particularly, the physic conditions such as temperature, humility, vibration and electro-magnetic interference etc are different. The period of field test depends on the laboratory simulation contents and the field conditions.

According to the development of rail transportation system and CBTC systems in the world, it is only the problem of time for the traditional train control system (track circuits based train control system) to be replaced by CBTC systems. At present, CBTC systems have been applied in light rail network and underground systems in the world. Particularly, when the signaling system is upgraded and the new line is constructed, CBTC is their first choice for train control systems. For example, the New York Metro selected Siemens's CBTC and Shanghai Metro selected Alstom's CBTC systems. It is also clear that $\mathrm{CBTC}$ will be applied in main line railway network. In Europe, ETCS level 2 has been in trial since 2000 [5].

It is true that transit from traditional train control system to CBTC systems is a long process. It is not possible for the transit to be completed in the short time since the cost and other reasons. However, from the above CBTC system analysis, it can be seen that the transit from the traditional system to CBTC system is very easy. This is one of the biggest advantages of CBTC systems since that can overlap on the traditional systems. Under the certain conditions, the two systems can coexist without interference. Before the CBTC is adjusted into normal operation, the old system can still be used. The traditional system can be removed only after the CBTC is in normal operation.

Since the CBTC system is modular system, the part of the traditional system can remain in the CBTC system. For example, as a relatively independent subsystem, the interlocking system can still be used in the CBTC system as long as the interface is upgraded. Therefore, when signaling system needs to be upgraded, CBTC can be implemented step by step.

\section{Conclusion}

It is very clear that CBTC system will replace the traditional train control system in railway network in the world. Its configuration, interfaces and dada format 
should be standardized. Its key technical issues are in common. As long as the standardizations can be observed, the CBTC systems manufactured by the different companies can be compatible on railway network. Moreover, CBTC system can be easily upgraded with development of new technologies in the key technical areas. Meanwhile, it is also easier for the traditional train control system to be transited into CBTC systems.

\section{References}

[1] Bin Ning " ETCS and CTCS “ Computers in Railways IX WIT Press pp 262 - 272 May 2004, Comprail 2004, Drensdon, Germany.

[2] Bin Ning "A comprehensive Information System for Railway Network" Computers in Railways VIII, WIT Press, pp 152 - 162, June 2002, Comprail 2002, Lemnos, Greece.

[3] Bin Ning "Intelligent Railway System in the $21^{\text {st }}$ Century" Computers in Railways VII, WIT Press, pp 1153 - 1163, September, 2000, Comprail 2000, Bologna, Italy.

[4] Bin Ning, K.-P. Li and Z.-Y. Gao "Modeling Fixed-Block Railway Signaling System Using Cellular Automata Model” International Journal Of Modern Physics C, Volume 16, Number 11 November 2005 pp 1793 1801.

[5] Eddie Goddard "London Underground Signaling Renewal" IRSE News Issue 111, February 2006. pp $1-7$.

[6] Rail Safety and Standards Board "Engineering Safety Management" (the "Yellow Book"). 